\title{
Flare observation of the Sun as a star by SUMER/SOHO in the hydrogen Lyman continuum ${ }^{\star}$
}

\author{
P. Lemaire ${ }^{1}$, P. Gouttebroze ${ }^{1}$, J.-C. Vial ${ }^{1}$, W. Curdt ${ }^{2}$, U. Schühle ${ }^{2}$, and K. Wilhelm ${ }^{2}$ \\ ${ }^{1}$ Institut d'Astrophysique Spatiale (IAS), Unité mixte CNRS-Université, Université de Paris XI, Bât. 121, 91405 Orsay, France \\ 2 Max-Planck-Institut für Aeronomie, 37191 Katlenburg-Lindau, Germany
}

Received 26 September 2003 / Accepted 4 February 2004

\begin{abstract}
During the execution of the programme "Sun as a star", while the SUMER (Solar Ultraviolet Emission of Emitted Radiation)/SOHO (SOlar and Heliospheric Observatory) slit was collecting the scattered radiation from the telescope mirror far away from the solar disk image, a class X5.3/3b flare erupted on the solar disk, on 25 August 2001. During the first phase of the flare a relative increase of a few percent was detected at the head of the hydrogen Lyman continuum. After correction from the instrumental parameters, the relative signal increase is $70 \%$ at the head of the Lyman continuum (910 ̊), and $190 \%$ in the C II $904 \AA$ multiplet. Accounting for the area of the flare region, the local increase of the radiance of the Lyman continuum and of the C II lines is estimated to be a factor of several thousands. We compare this result with other solar observations and models.
\end{abstract}

Key words. Sun: flares - Sun: UV radiation - stars: flare

\section{Introduction}

Only a few published papers have reported observations of the increase and shape of the solar spectrum in the ultraviolet (UV) during the impulsive phase of a flare and the time immediately following such an event. This is caused by the difficulty having the spectrometer slit at the exact location at the exact time of the beginning of a flare. There are also very few reports of the detection of solar flares in the UV spectrum of the integrated Sun (the Sun as a star). If the flare intensity is large enough, a modification in the disk-integrated solar UV flux can be detected, and, with the knowledge (e.g., from ground based observations) of the relative flare area, the local modification of the spectrum can be deduced to help the understanding of the global flare range.

Before the SKYLAB launch, few flare observations that included the hydrogen Lyman continuum ( $\mathrm{H} \mathrm{I} \mathrm{Lc)} \mathrm{were}$ made (see Kelly et al. 1972; Wood et al. 1972; Wood \& Noyes 1972). Later the SKYLAB/HCO spectroheliometer recorded several H I Lc spectra (Machado \& Noyes 1978), and semi-empirical models of flares have been built (Machado et al. 1980). Some flare observations down to H I Ly $\beta$ $(1025 \AA)$ have been performed with the OSO-8 multichannel spectrometer (Lemaire et al. 1984). A successful rocket launch

Send offprint requests to: $\mathrm{P}$. Lemaire,

e-mail: lemaire@ias.u-psud.fr

* Appendix A is only available in electronic form at http://www. edpsciences.org during a flare (Hirayama et al. 1985) of a spectrometer with high angular resolution has provided a map of the flare area near the head of H I Lc (880 ̊̊). Since then, the SUMER spectrometer launched on SOHO in 1995 is the first one to record this part of the spectrum spatially resolved.

Some flare observations in H I Lc have been performed with the full Sun integration (Sun as a star) and have been reported by Hall (1971). A large flare was detected by the SOLar-STellar Irradiance Comparison Experiment (SOLSTICE) on the Upper Atmospheric Research Satellite (UARS) (Brekke et al. 1996), but the data covered only the wavelength range above $1200 \AA$ A.

In this paper we present the results obtained from observations near the head of H I Lc of the Sun seen as a star during a X5.3/3b flare. The spectral resolution of SUMER allows us to extract information on the EUV continuum as well as on a large number of EUV lines After a brief description of the SUMER spectrometer parameters needed to describe the special operation used during the programme of the Sun seen as a star, and to understand the required data processing, we present the data and the corrections applied to get the true spectrum of the relative solar irradiance during the flare. We then compare the SUMER flare observations with data taken by ground and space instruments and we derive an estimate of the duration and the area of the flare during the impulsive phase. From the area of the flare we deduce the local radiance of the flare. The results are then compared with previous results and models. 


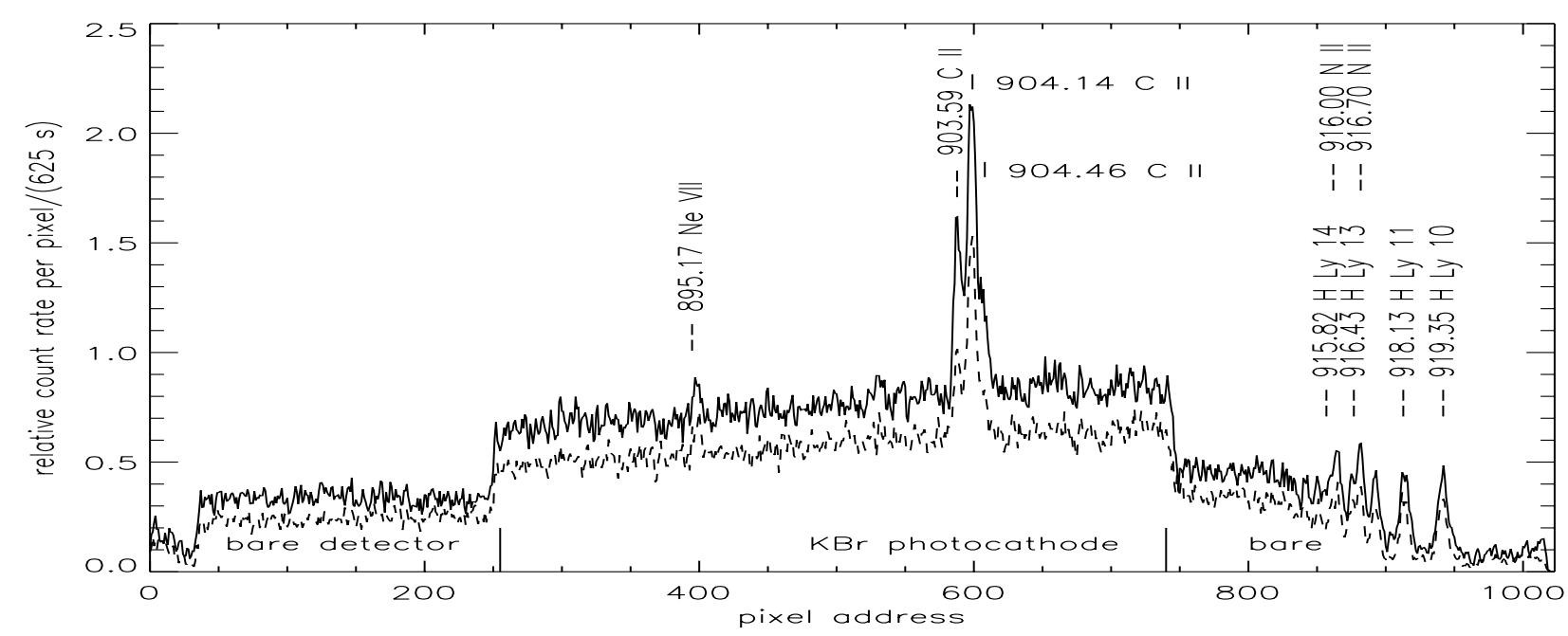

Fig. 1. Raw spectrum at the head of the Hi Lyman continuum. Data obtained before the flare: dashed line, during the flare: solid line. Wavelengths of lines are in $\AA$. Along the $x$ axis detector pixels 260 to 740 under the $\mathrm{KBr}$ photocathode are more sensitive than other pixels corresponding to the bare microchannel plate.

\section{Observations}

\subsection{SUMER observations}

The SUMER spectrometer on SOHO, located at the $L 1$ Lagrangian position, has been described in Wilhelm et al. (1995) and the in-flight performances are given in Wilhelm et al. (1997a) and Lemaire et al. (1997). The solar image is formed at the focus of the off-axis paraboloid telescope mirror where the spectrometer entrance slit is located. Then the beam issued from the slit is collimated, deflected at variable incidence by a plane mirror, diffracted and focussed by a concave grating onto a cross-delay-line detector $(1024$ spectral $\times$ 360 spatial pixels, Siegmund et al. 1995). On the A detector used during this observation the whole 800 to $1600 \AA$ (first grating order) spectral range can be swept by $44 \AA$ slices with an average of $0.044 \AA$ per pixel along the dispersion direction (the stigmatic spectra providing a monochromatic slit image with about one second of arc per pixel). During the observation, the 4 " width slit used provides a $0.18 \AA$ spectral resolution.

The SUMER telescope mirror is able to rotate around its focus in axes corresponding to solar $X$ and $Y$ ( $Y$ is along the polar axis) to select (or to scan) any position within a $\pm 32^{\prime}$ field of view.

During the 25 August 2001 16:30 UTC flare impulse, the SUMER slit was located at solar $X=860^{\prime \prime}$ and $Y=1700^{\prime \prime}$ (north) in SOHO coordinates (to be multiplied by 0.99 to obtain solar coordinates seen from Earth). The running programme was performed to obtain an average spectrum of the integrated Sun, by making use of the spatial smoothing resulting from the telescope scattered radiation with $8 \times 625 \mathrm{~s}$ exposures every $20 \AA$. The X5.3 flare erupted in the southern hemisphere in the NOAA 9591 active region at the SOHO solar coordinates $X=$ $-560^{\prime \prime}$ and $Y=-413^{\prime \prime}$ during a SUMER exposure centered on $902 \AA$ wavelength. The detector raw data, averaged along the slit, before and during the impulsive phase of the flare are displayed in Fig. 1. A significant increase of the signal is seen in the H I Lc and in all the lines within the $44 \AA$ spectral range

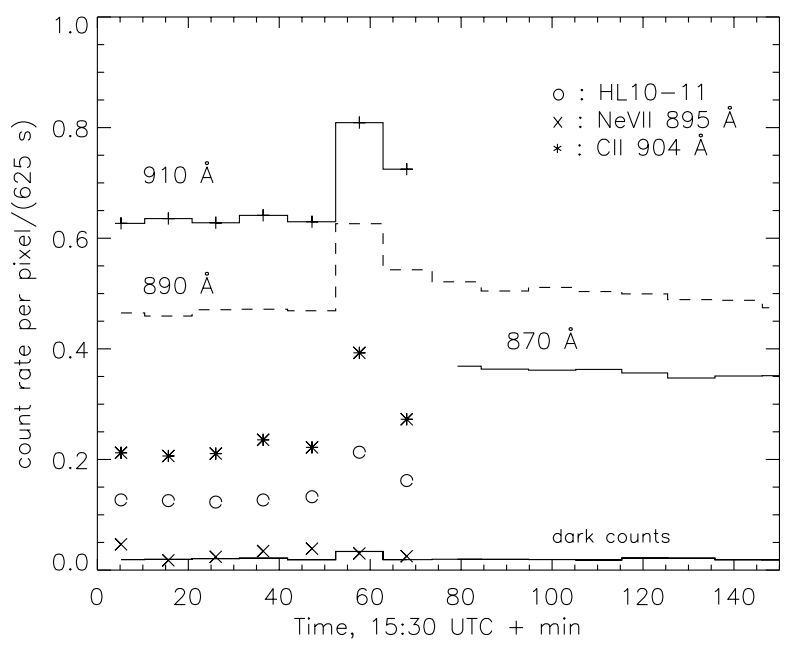

Fig. 2. Temporal evolution of the SUMER detector A counting rate in the continuum ( $910 \AA, 890 \AA, 870 \AA, 850 \AA$ ) and in the lines

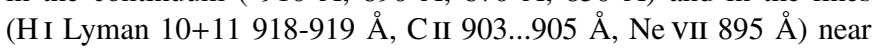
the head of the H I Lyman continuum.

covered. The corresponding temporal variations of the signal are shown in Fig. 2. The $890 \AA$ band is detected in two series of exposures where the wavelength has been shifted by $20 \AA$. During the $625 \mathrm{~s}$ exposure time correlated with the impulsive phase of the flare, the fractional increases of the signal ranged from $28 \%$ in $910 \AA \mathrm{H} \mathrm{I} \mathrm{Lc} \mathrm{to} 81 \%$ in the $904 \AA \mathrm{C}$ II lines. But these relative increases do not correspond to the real increases of solar irradiance values (or the Sun seen as a star) because the scattered-radiation contributions of different areas of the solar disk have different weights at the location of the slit in relation to the distance between the emission and the slit location.

The modelling of the telescope scatter properties can be done from the pre-launch measurements of the telescope point spread function (PSF) and its far-wing extension made by Saha \& Leviton (1993). The convolution by a flat solar disk gives a good estimate of the scattered radiation that compares 


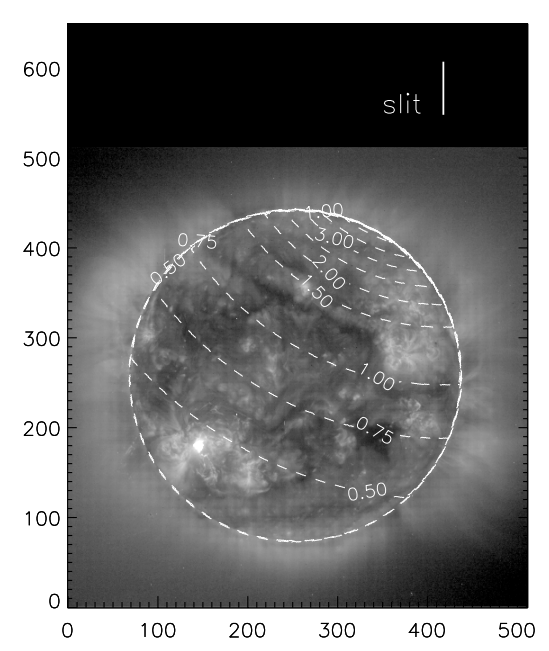

Fig. 3. Relative contributions of the solar disk scatter to the signal collected by the $4^{\prime \prime}$ slit located at solar $X=860^{\prime \prime}$ and $Y=1700^{\prime \prime}$. The Fe XII $195 \AA$ AIT image taken during the flare is superposed. The scales of abcissa and ordinate are in EIT pixels $\left(2.56^{\prime \prime}\right)$ (courtesy of the EIT team). Labels $(0.50,0.75, \ldots)$ indicate the local contribution to the observed signal from a flat solar disk of unity value.

Table 1. Irradiance variation of the solar flux in the vicinity of $900 \AA$ A (H I Lyman continuum) during the X5.3/3b flare of 25 August 2001.

\begin{tabular}{lcccc}
\hline \hline & \multicolumn{2}{c}{ Raw } & \multicolumn{2}{c}{ Corrected } \\
\hline & $I_{\text {before }}$ & $I_{\text {flare }}$ & $I_{\text {flare }}-I_{\text {before }}$ & $I_{\text {flare }} / I_{\text {before }}$ \\
& \multicolumn{3}{c}{ count rate per pixel $/(625 \mathrm{~s})$} \\
\hline $910 \AA$ & 0.632 & 0.809 & 0.421 & $1.7 \pm 0.2$ \\
$\left(\mathrm{Lc}_{\text {head }}\right)$ & & & & \\
$890 \AA$ & 0.467 & 0.626 & 0.378 & $1.8 \pm 0.2$ \\
$\mathrm{C}$ II & 0.217 & 0.393 & 0.419 & $2.9 \pm 0.3$ \\
H I L10-11 & 0.127 & 0.213 & 0.205 & $2.6 \pm 0.3$ \\
\hline
\end{tabular}

favourably with the observations within relative deviations of $30 \%$. From this model we can deduce the contribution of each part of the Sun at a given distance from the limb. For the flare observations the results are shown in Fig. 3. The Fe XII $195 \AA$ EIT image taken during the flare is superposed.

At the flare location the local weight is only 0.42 with respect to disk center, so the measured flare increase must be corrected accordingly:

$\left(I_{\text {flare }}-I_{\text {before }}\right)_{\text {corrected }}=\left(I_{\text {flare }}-I_{\text {before }}\right) / 0.42$,

where $I_{\text {flare }}$ and $I_{\text {before }}$ are the real relative irradiances during and before the flare.

The results give the irradiance ratios reported in Table 1, with relative increases of $72 \%$ at $910 \AA$, (Lyman continuum head) and $193 \%$ in the C II lines as the extreme values. The error on the local weight (smaller than 10\%) is determined from a simulation of the increase of the PSF core width by $50 \%$ and the wing contribution by $50 \%$. The very small contribution of the statistical error in the counts integrated over $1 \AA$ (or over the lines) and the exposure time are also taken into account in the error value in the $I_{\text {flare }} / I_{\text {before }}$ ratio given in Table 1 .

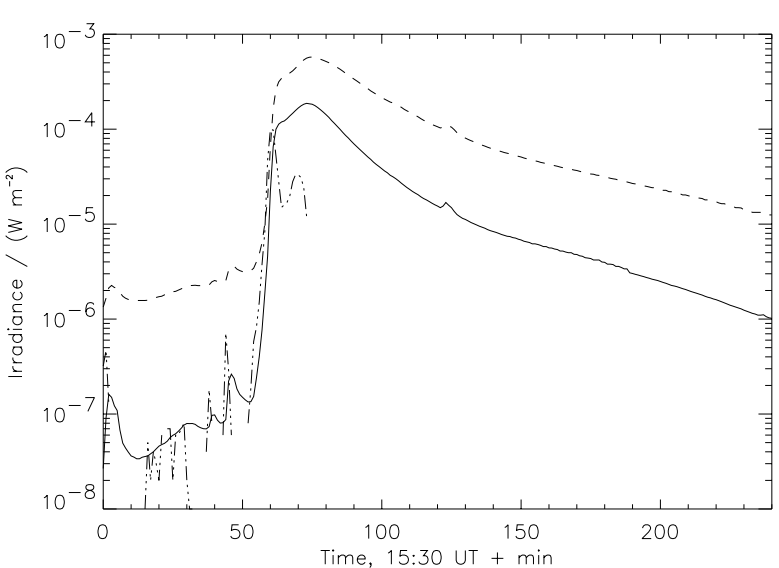

Fig. 4. Variation of the $X$-ray flux at Earth during the flare measured in the two channels of GOES $10(0.5$ to $4 \AA$, and 1 to $8 \AA)$. The (- - - - ) line is the time derivative of the 0.5 to $4 \AA \mathrm{X}$-ray flux (unit, $\mathrm{W} \mathrm{m}^{-2} \mathrm{~min}^{-1}$ ).

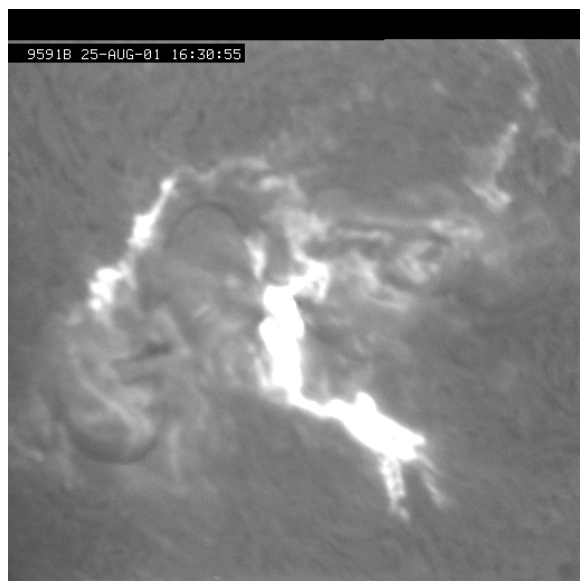

Fig. 5. H $\alpha$ spectroheliogram recorded by Holloman AFB (Air Force Base) Observatory at the time of maximum area of detector saturation during the $\mathrm{X} 5.3 / 3 \mathrm{~b}$ flare.

\subsection{X-rays and $\mathrm{H} \alpha$ observations}

The X5.3 flare was detected in the two channels ( 0.5 to $4 \AA$, and 1 to $8 \AA$ ) of the GOES 10 satellite. The variation of the irradiance is shown in Fig. 4. After a few oscillations, the X-ray flux increases by several decades in a few minutes and then slowly decreases during several hours. The plot of the time derivative of the short wavelength X-ray flux variation shows the dramatic change of the X-ray emission.

The ground-based Holloman solar observatory has made observations of the flare area with a high time resolution in the $\mathrm{H} \alpha$ line (an image every 1 to $5 \mathrm{~min}$ ). Figure 5 shows the spectroheliogram taken when there is a maximum of saturated pixels above the relative digital number (DN) of 254 (plages are in the 170 to $230 \mathrm{DN}$ range, and the average quiet Sun is $130 \mathrm{DN})$. Using this set of images we have established the variation of this number (or relative area) as a function of the flare evolution (Fig. 6).

In the same figure the time derivative of the GOES 1 to $8 \AA$ $\mathrm{X}$-ray emission of Fig. 4 is superimposed. There is a good time correlation between the soft X-ray emission and the $\mathrm{H} \alpha$ area at the 1 min sampling. Applying the procedure of 


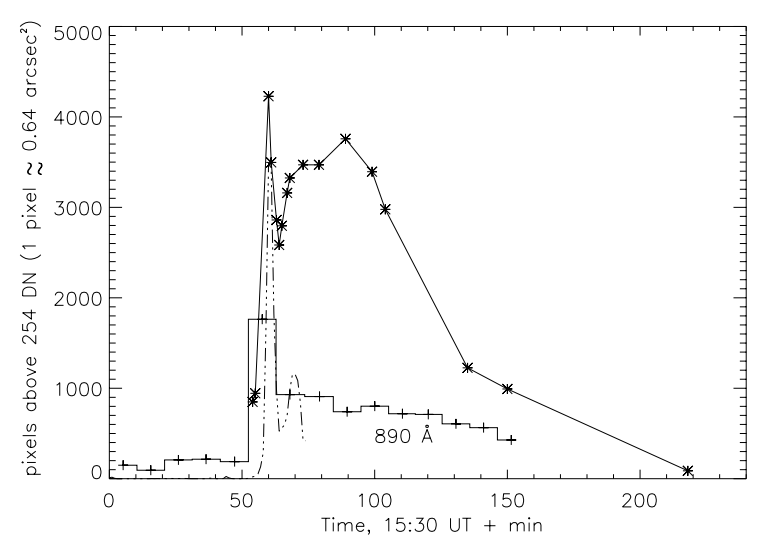

Fig. 6. Evolution of the number of saturated pixels in the series of $\mathrm{H} \alpha$ spectroheliogram recorded by Holloman AFB Observatory during the flare (-*-). The relative variation of the $\mathrm{X}$-ray flux derivative is scaled and superposed (- - - -). The relative variation of the $890 \AA$ A signal (arbitrary scale) is overplotted; each -+- label corresponds to an exposure time.

Thomas et al. (1985) to the GOES 10 channel data, we estimate the temperature of the flare to be $\approx 18 \times 10^{6} \mathrm{~K}$ and the volumetric emission measure to be $\approx 4 \times 10^{49} \mathrm{~cm}^{-3}$ from their Eqs. (9)-(11).

To obtain the flare emission, several correction factors are required:

- at the apparent maximum of the $\mathrm{H} \alpha$ area during the flare the number of $\mathrm{H} \alpha$ saturated pixels is 4288 . As each pixel has a size of $\approx 0.64 \operatorname{arcsec}^{2}$, this corresponds to an area of $a_{\text {max }} \approx 2744 \operatorname{arcsec}^{2}$ in the plane of the sky (the pixel is measured by comparison with the $\mathrm{H} \alpha$ Holloman full disk image taken at 16:41 UTC);

- at the time of the observation, before the flare, several plages contribute to the flux into the slit. To determine the real flare to quiet-Sun (QS) contribution we have measured the area of each plage and weighted it by the contribution factor to scattered light (as shown in Fig. 3). The obtained relative plage area represents $2.5 \%$ of the solar disk area. To make an estimate of the real quiet-Sun (QS) contribution to the signal at the head of H I Lc we use the average plage to quiet-Sun ratio (4.9) derived from Vernazza \& Reeves (1978) measurements. If $I_{\mathrm{P}}$ is the relative plage contribution to the irradiance,

$\left(I_{\mathrm{P}}+I_{\mathrm{QS}}\right) / I_{\mathrm{QS}} \approx 4.9$

then, scaling to the solar surface,

$0.025\left(I_{\mathrm{P}}+I_{\mathrm{QS}}\right)+0.975 I_{\mathrm{QS}}=1$

and $I_{\mathrm{QS}}=0.91$ of the observed emission;

- the flare was observed at $\vartheta=46.5^{\circ}(\vartheta$ is the heliocentric angle from the center of the disk). The corrected area used is given by $A_{\text {corr }}=a_{\max } / \cos \vartheta \approx 3987 \mathrm{arcsec}^{2}$. The real surface $\left(S_{\text {corr }}\right)$ on the solar sphere, corrected for the projection effect and normalized to the visible hemisphere surface is $6.9 \times 10^{-4}$, within the range of a $3 \mathrm{~b}$ flare ( 6 to $12 \times 10^{-4}$ of hemisphere). The ratio $r_{\mathrm{av}}$ between the flare and the average solar radiance emission is $\approx 1000$
Table 2. Relative local variation of the flare $\left(I_{\mathrm{flare}}\right)$ to quiet solar $\left(I_{\mathrm{QS}}\right)$ emission in the vicinity of $900 \AA$ (H I Lyman continuum) during the X5.3/3b flare of 25 August 2001.

\begin{tabular}{lccc}
\hline \hline & $I_{(\mathrm{P}+\mathrm{QS})} / I_{\mathrm{QS}}$ & QS contribution & $I_{\text {flare }} / I_{\mathrm{QS}}$ \\
$910 \AA$ & 4.9 & 0.91 & $\geq 1.1 \pm 0.1 \times 10^{3}$ \\
$\left(\mathrm{Lc}_{\text {head }}\right)$ & & & \\
$890 \AA$ & 4.7 & 0.91 & $\geq 1.3 \pm 0.1 \times 10^{3}$ \\
$\mathrm{C}_{\text {II }}$ & 2.2 & 0.97 & $\geq 2.8 \pm 0.3 \times 10^{3}$ \\
$\mathrm{H} \mathrm{I} \mathrm{I} 10-11$ & 4.3 & 0.92 & $\geq 2.5 \pm 0.2 \times 10^{3}$ \\
\hline
\end{tabular}

(from the $1+r_{\mathrm{av}} S_{\mathrm{corr}}=1.7$ ), and 1100 \pm 200 (from 1000/0.91 ratio) between the flare and the quiet Sun radiance at the head of the H I Lc.

A similar set of corrections can be applied to the lines, and the results are given in Table 2 .

As mentioned before, the exposure time was $625 \mathrm{~s}$, which means some temporal averaging during the flare. The temporal correction factor will be reviewed in the general discussion.

\section{Comparison with flare model predictions}

For a comparison with the observations we use:

- the VAL C (Vernazza et al. 1981) model as a quiet Sun reference model;

- the plage P1 (Lemaire et al. 1981);

- the MAVN F2 (bright) and F1 (faint) (Macchado et al. 1980) flare models.

It should be noted that in all these models a static onedimensionally stratified atmosphere is assumed.

These semi-empirical models can be used to calculate the H I Lyman lines and continuum radiances and to compare them to those observed ones. The computation was performed with the latest version of the stellar atmosphere programme HYDR (the previous version was used for modelling the chromosphere of A-type stars: see Gouttebroze et al. 1999). The model hydrogen atom includes 20 levels and a continuum. The radiative transfer equations are solved for 66 lines and five continua. Other transitions are set in radiative balance. Effects of partial frequency redistribution are taken into account for the $\mathrm{L} \alpha$ and $\mathrm{L} \beta$ lines.

The results at the head of H I Lc are shown in Fig. 7. From VAL $\mathrm{C}$ to MAVN F2 the increase of radiance is a factor of $\approx 3 \times 10^{3}$ while the observations give $\geq 1.1 \times 10^{3}$. The slope of the continuum decreases with activity level. The results of the computation for some hydrogen lines are given in Table 3. The $\mathrm{HI} L \alpha$ and $\mathrm{L} \beta$ line ratio (MAVN F2 to VAL C) is smaller than that of the continuum, but in the higher transitions of the Lyman series where the plasma becomes optically thin in the corresponding lines this ratio is larger.

\section{Discussion}

\subsection{Accuracy of the flare emission evaluation}

The SUMER slit was at $0.9 R_{\odot}$ (solar radius) above the solar limb collecting the telescope scattered radiation averaged over 


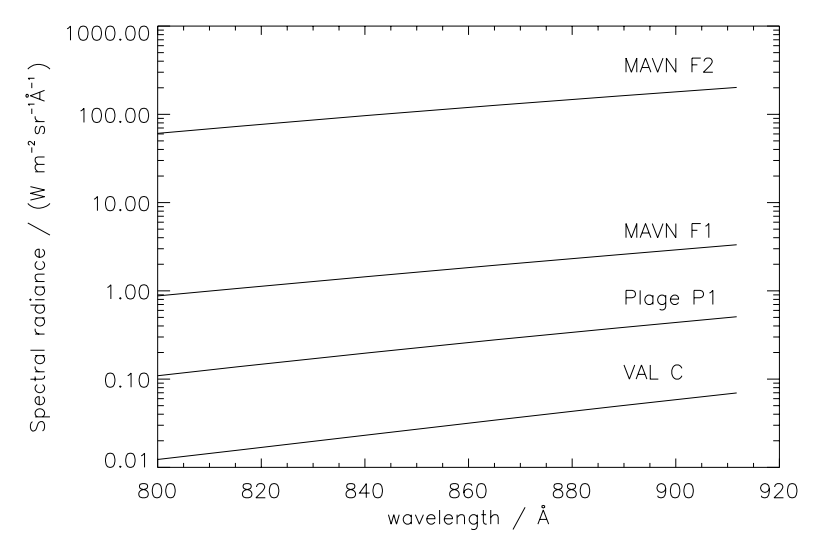

Fig. 7. Model predictions of H I Lc radiance: quiet Sun (VAL C), plage (P1), flare (MAVN F1 and F2).

Table 3. Comparison of model predictions to observed values near $900 \AA$ (H I Lyman continuum) during the X5.3/3b flare of 25 August 2001.

\begin{tabular}{lcccc}
\hline \hline \multicolumn{4}{c}{$\mathrm{W} \mathrm{m}^{-2} \mathrm{sr}^{-1} \AA^{-1}$} & \multicolumn{2}{c}{ flare/QS } \\
\hline VALC & MAVNF2 & Computed & Observation \\
$910 \AA$ & $7.0 \times 10^{-2}$ & $2.0 \times 10^{2}$ & $2.8 \times 10^{3}$ & $\geq 1.1 \times 10^{3}$ \\
$\left(\mathrm{Lc}_{\text {head }}\right)$ & & & & \\
$890 \AA$ & $4.9 \times 10^{-2}$ & $1.6 \times 10^{2}$ & $3.2 \times 10^{3}$ & $\geq 1.3 \times 10^{3}$ \\
C II & & & & $\geq 2.6 \times 10^{3}$ \\
H I L10-11 & $1.8 \times 10^{1}$ & $8.4 \times 10^{4}$ & $4.6 \times 10^{3}$ & $\geq 2.5 \times 10^{3}$ \\
H I L $\alpha$ & $1.0 \times 10^{4}$ & $3.5 \times 10^{6}$ & $3.5 \times 10^{2}$ & \\
H I L $\beta$ & $2.4 \times 10^{2}$ & $6.6 \times 10^{5}$ & $2.7 \times 10^{3}$ & \\
\hline
\end{tabular}

the solar disk. The data are corrected for the local contribution at this position; the relative uncertainty of this correction is estimated to be within $10 \%$ (see Sect. 2.1).

The flare area is computed from $\mathrm{H} \alpha$ spectroheliograms. The determination of the area using the maximum number of pixels at saturation level in the image probably overestimates the true flare area (wings of the point spread function, leakage between pixels, etc). The standard Sawyer (1967) formula used to compute the flare area, $A_{\text {corr }}=a_{\max } /(\cos \vartheta+0.2 \sin \vartheta)$ would have given $3293 \operatorname{arcsec}^{2}$, which reduces the area by 1.2 and increases the flare-to-quiet-Sun radiance ratio by the same factor. There is also an uncertainty in the exact time of the $\mathrm{H} \alpha$ flare maximum. The result must be an overestimate of the maximum area emission of the flare by a factor one to two or larger.

The $625 \mathrm{~s}$ exposure time includes the rising phase from preflare to flare $\mathrm{H} \alpha$ emission, the maximum, and the beginning of the declining phase; more discussion will be given in the following section. The real maximum emission can be at least 3 to 10 times higher (for a 1 to 3 min duration) than the value obtained during the averaging exposure time.

\subsection{Comparison with computations and observations}

\subsubsection{Flare-to-quiet-Sun ratio}

The flare to quiet-Sun emission ratio can be near to $10^{4}$ for this X5.3 flare in the high terms of the H I Lyman series, in the $\mathrm{HI}$ Lc, and also in C II lines.
With a typical $0.055 \mathrm{~W} \mathrm{~m}^{-2} \mathrm{sr}^{-1} \AA^{-1}$ quiet Sun radiance at the head of HI Lc (see Appendix A), at the maximum of the flare we can obtain a few $10^{2} \mathrm{~W} \mathrm{~m}^{-2} \mathrm{sr}^{-1} \AA^{-1}$. This value is equivalent to or higher than the MAVN F2 prediction. This computation was done to represent the $-\mathrm{N}$ flare observed on 2 September 1973 by the HCO/SKYLAB spectrometer (Machado et al. 1980). The $\mathrm{H} \alpha$ class -N flare, corresponding to an area smaller than $1 \times 10^{-4}$ hemisphere, was analyzed by the $5^{\prime \prime} \times 5^{\prime \prime}$ entrance hole of the spectrometer. This flare produced the highest HI Lc measured by this instrument, with $7.8 \times 10^{3} \mathrm{~W} \mathrm{~m}^{-2}$ total H I Lc flux (Machado \& Noyes $1978)$ and $1.82 \times 10^{1} \mathrm{~W} \mathrm{~m}^{-2} \mathrm{sr}^{-1} \AA^{-1}$ radiance at the continuum head (902 $\AA$ ). This value is four times lower than the most conservative value estimated from our observation.

In the C II $904 \AA$ lines, the lower estimate of the radiance increase is $2.6 \times 10^{3}$. For comparison, the estimate for the C II $1336 \AA$ lines increase in a X3/3b class flare (Brekke et al. 1996) is $3.4 \times 10^{3}$.

From an analysis of several flares, Hall (1971) determines a $(2.4 \pm 1.6)$ min rise time and $(4.4 \pm 3.0)$ min decay time for lines in the H I Lyman series. Similar values (1.4 min rise time and greater than 5 min decay time) are reported by Wood \& Noyes (1972) at the head of H I Lc. The time delay between several chromospheric lines has been measured during the observation of the Sb/1b 15 April, 1978 (08:00-08:58 UTC) flare done by the LPSP-OSO-8 experiment (Lemaire et al. 1984) using a $1^{\prime \prime} \times 10^{\prime \prime}$ entrance slit. The H I L $\alpha$ line peaks first $\left(T_{0}\right)$, then the $\mathrm{H} \mathrm{I} \mathrm{L} \beta$ and $2800 \AA \mathrm{Mg}$ II lines peak at $T_{0}+20 \mathrm{~s}$, and the $\mathrm{H} \epsilon$ and $3950 \AA$ Ca II lines at $T_{0}+40 \mathrm{~s}$. Wood \& Noyes (1972) also note that the H I Lyman line intensities peak $\approx 2$ min before the $\mathrm{H} \alpha$ line intensity (see also Hall 1971).

Taking into account this flare evolution, and using the $\mathrm{H} \alpha$ timing as a reference, the flare burst in $\mathrm{H} \mathrm{I} \mathrm{Lc} \mathrm{starts} \mathrm{about} 5 \mathrm{~min}$ after the beginning of the $625 \mathrm{~s}$ exposure time. Only a small part of the exposure time is filled by the maximum of the flare. The $\mathrm{H} \alpha$ flare area evolution shows a sharp peak followed by a succession of bursts localized near the first location, with a smoothed envelope which decreases $(1 / \mathrm{e})$ in $70 \mathrm{~min}$. Within $2 \times 625$ s exposure times the H I Lc and the C II and H I L1011 lines have decayed by $1 / \mathrm{e}$, much faster than $\mathrm{H} \alpha$.

\subsubsection{Physical characteristics of the flare}

To interpret the H I Lc data, an Eddington-Barbier relation can be used as a first approximation to derive some physical characteristics of the atmosphere at the level where the emission originates. If $b_{1}$ is the departure coefficient from the local thermodynamic equilibrium (LTE) of the first level of hydrogen, $\tau$ the optical depth, $B_{\lambda}$ the Planck function, $T_{\mathrm{c}}$ the colour temperature of the observed continuum emission, $L_{\lambda}$ the spectral radiance, and $\mu$ the cosine of the emergence angle, then we have the following formula (Machado \& Noyes 1978):

$b_{1}(\tau=1)=B_{\lambda}\left(T_{\mathrm{c}}\right) / L_{\lambda}(\mu=1)$.

With the radiance measured at two wavelengths (890 $\AA$ and $910 \AA)$ and the same $b_{1}(\tau=1)$ and $T_{\mathrm{c}}$ we can derive the two quantities. A decrease of $b_{1}$ towards unity indicates that the local atmosphere is approaching thermodynamic equilibrium. 
The $b_{1}$ and $T_{\mathrm{c}}$ results are very sensitive to the accuracy of the $L_{\lambda}(910 \AA) / L_{\lambda}(890 \AA)$ ratio and of the absolute value of each spectral radiance. The quiet-Sun H I Lc reference is discussed in Appendix A. The average detector $B$ values give $T_{\mathrm{c}}=9000 \mathrm{~K}$ and $b_{1} \approx 900$ with the $55 \mathrm{~mW} \mathrm{~m}^{-2} \mathrm{sr}^{-1} \AA$ radiance value at the head of H I Lc. These values are similar to the values obtained from HCO/SKYLAB data (Vernazza \& Reeves 1978; Chapman 1981 Table 3.5, and Vernazza et al. 1981 Table 8 and Fig. 31) (cf. Appendix A). Nevertheless $T_{\mathrm{c}}$ and $b_{1}$ experience a rapid variation with optical depth and the values must be taken cautiously (Vernazza et al. 1981 Sect. V).

Applying the $890 \AA$ and $910 \AA$ correction factors of Table 3 for the flare observation, one obtains $T_{\mathrm{c}}=12150 \mathrm{~K}$ and $b_{1}=$ 48. An increase of 10 in the correction factor will modify the temperature by a few thousand $\mathrm{K}$ and reduce $b_{1}$ by a significant factor (up to ten). For intense flares, the departure coefficient of the first level of hydrogen is near to 1 ; in the flare the local atmosphere is near LTE.

A coarse estimate of the electronic density $n_{\mathrm{e}}$ and the population $N_{1}$ is obtained from the quantum number of the lastresolvable Lyman line (De Feiter \& Švestka 1975). With a quantum number higher than 14 in this case, $n_{\mathrm{e}}$ must be smaller than $10^{13} \mathrm{~cm}^{-3}$ and $N_{1}$ smaller than $10^{20} \mathrm{~cm}^{-3}$. In our flare data, the result of the integration on the full disk and the observations at the beginning of the flare are a mixing of pre-flare and flare observations diluted in the plages and quiet-Sun contributions. The determination of the electronic density $n_{\mathrm{e}}$ and the population $N_{1}$ from the quantum number of the last-resolvable Lyman line has to be taken with caution.

\section{Conclusion}

1. Using the scattered radiation of the SUMER telescope mirror we have obtained the first high-spectral resolution observation at the head of the hydrogen Lyman continuum of a solar flare seen as a star flare.

2. The Sun-as-a-star irradiance is multiplied by factors of 1.7, 2.9, and 2.6 at HI Lc head $910 \AA$, C II $904 \AA$ and H I L10-11 lines during the X5.3/3b flare.

3. The local radiance enhancement is higher than $1 \times 10^{3}$ in the HI Lc, and in the C II and H I L10-11 lines.

4. To increase the accuracy in the determination of the physical parameters of a flare, data acquisition with high time resolution, combined with high angular resolution in a moderate field of view is required.

5. Such results in the emission from a solar flare could be of interest for observing and modelling of various flare stars such as dMe.
Acknowledgements. SOHO is a mission of international cooperation between ESA and NASA. The SUMER project is financially supported by DLR, CNES, NASA and ESA PRODEX programme (Swiss contribution). The Holloman $\mathrm{H} \alpha$ and GOES data were extracted from the Space Environment Center data base, contributed by the US Department of Commerce, NOAA. We also thank the EIT/SOHO consortium for the use of their EUV solar images. We thank the referee (Dr. H. Peter) for his helpful comments.

\section{References}

Brekke, P., Rottman, G. J., Fontenla, J., \& Judge, P. G. 1996, ApJ, 468, 418

Chapman, G. 1981, in Solar Active Regions, Skylab Solar Workshop III, ed. F. Q. Orrall (Boulder: Colorado Associated University Press), 43

Curdt, W., Brekke, P., Feldman, U., et al. 2001, A\&A, 375, 591

De Feiter, L. D., \& Švestka, Z. 1975, Sol. Phys., 41, 415

Fontenla, J. M., Avrett, E. H., \& Loser, R. 1993, ApJ, 406, 319

Gouttebroze, P., Freire Ferrero, R., Marilli, E., \& Catalano, S. 1999, A\&A, 348, 198

Hall, L. A. 1971, Sol. Phys., 21, 167

Hirayama, T., Tanaka, K., Watanabe, T., et al. 1985, Sol. Phys., 95, 281

Hollandt, J., Schühle, U., Paustian, W., et al. 1996, Appl. Opt., 35, 5125

Kelly, P. T., \& Rense, W. A. 1972, Sol. Phys., 26, 431

Lemaire, P., Choucq-Bruston, M., \& Vial, J.-C. 1984, Sol. Phys., 90, 63

Lemaire, P., Gouttebroze, P., Vial, J.-C., \& Artzner, G. E. 1981, A\&A, 103,160

Lemaire, P., Wilhelm, K., Curdt, W., et al. 1997, Sol. Phys., 170, 105

Machado, M. E., \& Noyes, R. W. 1978, Sol. Phys., 59, 129

Machado, M. E., Avrett, E. H., Vernazza, J. E., \& Noyes, R. N. 1980, ApJ, 242, 336

Saha, T. T., \& Leviton, D. B. 1993, Proc. SPIE, 1494, 9

Sawyer, C. B. 1967, ApJ, 147, 193

Siegmund, O. H. W., Gummin, M. A., Sasseen, T., et al. 1995, Proc. SPIE, 2280, 89

Schühle, U., Curdt, W., Hollandt, J., et al. 2000, Appl. Opt., 39, 418

Thomas, R. J., Starr, R., \& Crannell, C. J. 1985, Sol. Phys., 95, 323

Vernazza, J. E., \& Reeves, E. M. 1978, ApJS, 37, 485

Vernazza, J. E., Avrett, E. H., \& Loeser, R. 1981, ApJS, 45, 635

Wilhelm, K., Curdt, W., Marsch, E., et al. 1995, Sol. Phys., 162, 189

Wilhelm, K., Lemaire, P., Curdt, W., et al. 1997a, Sol. Phys., 170, 75

Wilhelm, K., Lemaire, P., Feldman, U., et al. 1997b, Appl. Opt., 36, 6416

Wilhelm, K., \& Kalkofen, W. 2003, A\&A, 408, 1137

Wood, Jr, A. T., \& Noyes, R. W. 1972, Sol. Phys., 24, 180

Wood, Jr, A. T., Noyes, R. W., Dupree, A. K., et al. 1972, Sol. Phys., 24, 169 
P. Lemaire et al.: Flare observation of the Sun as a star by SUMER/SOHO, Online Material $p 1$

\section{Online Material}


P. Lemaire et al.: Flare observation of the Sun as a star by SUMER/SOHO, Online Material $p 2$

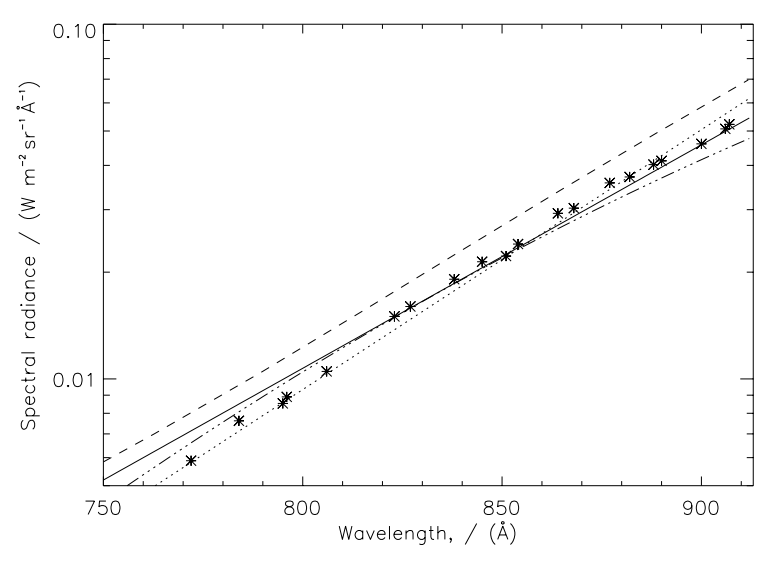

Fig. A.1. Average quiet-Sun H I Lc data from HCO/SKYLAB $(*)$. The dotted line represents the least-square fit of the data. Fits from models C of VAL81 (full line), of FAL93 (dashed/dotted line) and from our computation (dashed line) are shown.

\section{Appendix A: The quiet Sun hydrogen Lyman continuum}

The solar H I Lc (hydrogen Lyman continuum) has been observed since the beginning of the 1960s. The main observations have been made with the Harvard spectroheliographs on OSO-4, OSO-6 and SKYLAB/ATM. Since this last observation (1973) no other spectroheliograph on a spacecraft has provided the detailed spectra of this continuum until the SUMER observations.

The knowledge of the slope and the radiance of the H I Lc are essential parameters in the building of solar models where the hydrogen is the main ingredient. The slope of the continuum provides the colour temperature at the top of the chromospheric plateau, which can be combined with the radiance to determine the coefficient of departure from the local thermal equilibrium (LTE).

The HCO/SKYLAB H I Lc data for the average quiet Sun are plotted in Fig. A.1 (see Vernazza \& Reeves 1978; Vernazza et al. 1981). To fit these data several computations have been performed. The results from VAL81C (Vernazza et al. 1981) and FAL93C (Fontenla et al. 1993) models are shown in the same figure. Also, using the VAL81C model as input we have recomputed the $\mathrm{HI} \mathrm{Lc}$ and the result is given in Fig. A.1. The original VAL81C and our computation give a straight line in logarithmic scaling; the small discrepancy in absolute value and slope is related to the use of updated atomic data input in our computation. The FAL93C exhibits a small curvature; this can be the result of the introduction of a detailed treatment of particle diffusion and, as a consequence, an improved computation of the departures from local ionization equilibrium. In the 750 to $912 \AA \mathrm{H} \mathrm{I} \mathrm{Lc} \mathrm{range} \mathrm{the} \mathrm{discrepancy} \mathrm{is} \mathrm{still} \mathrm{small} \mathrm{and}$ we fit the $\mathrm{HCO}$ data by a straight line obtained from a leastsquare computation (see Fig. A.1); the slope can be used as a reference slope of the quiet-Sun H I Lc.

Hereafter we present the results of a set of quiet Sun observations made by SUMER. By comparison with SKYLAB/ATM observations, we are able to derive a range of temperatures for the Planck function fitting the data in the 800 to $900 \AA$ spectral range.
Table A.1. Quiet-Sun reference spectrum observations used to obtain the H I Lyman continuum.

\begin{tabular}{|c|c|c|c|c|c|}
\hline $\begin{array}{l}\text { Date } \\
\text { mmdd-hrmn }\end{array}$ & Slit & $\begin{array}{c}\text { Exposure } \\
\text { (s) }\end{array}$ & $\begin{array}{l}\text { Rotation } \\
\text { corrected }\end{array}$ & $\begin{array}{c}\text { Pointing } \\
x^{\prime \prime}, y^{\prime \prime}\end{array}$ & Detector \\
\hline \multicolumn{6}{|l|}{ year 1996} \\
\hline $0130-0457$ & 2 & 300 & no & 0,0 & A \\
\hline $0223-2134$ & 4 & 100 & yes & 1,0 & $\mathrm{~A}$ \\
\hline 0330-0141 & 4 & 100 & yes & 9,0 & A \\
\hline $0330-0400$ & 4 & 100 & yes & 31,0 & $\mathrm{~A}$ \\
\hline $0422-1835$ & 4 & 115 & yes & 3,0 & $\mathrm{~A}$ \\
\hline $0422-2104$ & 4 & 115 & yes & 3,0 & $\mathrm{~A}$ \\
\hline $0502-0125$ & 7 & 115 & yes & $-106,-120$ & A \\
\hline $0610-2140$ & 4 & 115 & yes & 111,762 & A \\
\hline 0617-1849 & 4 & 115 & yes & 3,0 & A \\
\hline 0727-0104 & 4 & 115 & yes & 3,0 & A \\
\hline 0801-1622 & 4 & 115 & yes & 3,0 & A \\
\hline 0801-1852 & 4 & 115 & yes & 3,0 & A \\
\hline 0812-0113 & 4 & 115 & yes & 3,0 & A \\
\hline 0826-1301 & 4 & 115 & yes & 3,0 & A \\
\hline $1011-0516$ & 4 & 115 & yes & 22,0 & B \\
\hline $1017-0532$ & 4 & 115 & no & 0,0 & B \\
\hline $1022-0131$ & 4 & 115 & no & 0,249 & B \\
\hline $1028-0357$ & 4 & 115 & no & 0,0 & B \\
\hline $1112-0947$ & 4 & 115 & no & 0,0 & B \\
\hline $1113-2336$ & 4 & 115 & no & 0,0 & B \\
\hline $1119-0010$ & 7 & 100 & no & $0,-40$ & B \\
\hline $1125-1625$ & 4 & 100 & no & 0,200 & B \\
\hline \multicolumn{6}{|l|}{ year 1997} \\
\hline 0116-0114 & 4 & 100 & no & 0,0 & $\mathrm{~B}$ \\
\hline 0222-0340 & 8 & 100 & no & 0,0 & B \\
\hline 0418-2001 & 4 & 100 & no & 0,0 & B \\
\hline 0420-0003 & 2 & 300 & yes & 0,0 & B \\
\hline \multicolumn{6}{|l|}{ year 1998} \\
\hline $0211-2233$ & 8 & 100 & no & $430,-300$ & B \\
\hline 0613-1631 & 4 & 100 & no & 0,0 & B \\
\hline & & $\begin{array}{l}\text { slit: } 2 \\
\text { slit: } 4 \\
\text { slit: } 7 \\
\text { slit: } 8\end{array}$ & $\begin{array}{r}1^{\prime \prime} \times 300^{\prime \prime} \\
1^{\prime \prime} \times 120^{\prime \prime} \\
0.3^{\prime \prime} \times 120^{\prime \prime} \\
0.3^{\prime \prime} \times 120^{\prime \prime}\end{array}$ & & \\
\hline
\end{tabular}

\section{A.1. Data}

Using the SUMER A detector several spectral scans of the full spectral range have been performed of quiet-Sun areas. For this study, 14 quiet-Sun spectra have been used to extract the H I Lc data. The selected spectral scans taken with the A detector are reported in Table A.1. For comparison 14 quiet-Sun spectral scans using the B detector have been added. Each reference spectrum observation is done by scanning the full spectral range ( 780 to $1610 \AA$ for the A detector or 670 to $1460 \AA$ for the $B$ detector) in increments, as noted in Table A.1. At each step the 1024 spectral pixels $(\approx 0.044 \AA$ per pixel) and either 120 or 360 angular pixels $\left(\approx 1^{\prime \prime}\right.$ per pixel) are recorded.

Each individual detector window is flat-fielded, corrected for deadtime and local pixel saturation following the usual SUMER procedures. Then, a normalized averaging along the 
P. Lemaire et al.: Flare observation of the Sun as a star by SUMER/SOHO, Online Material p 3

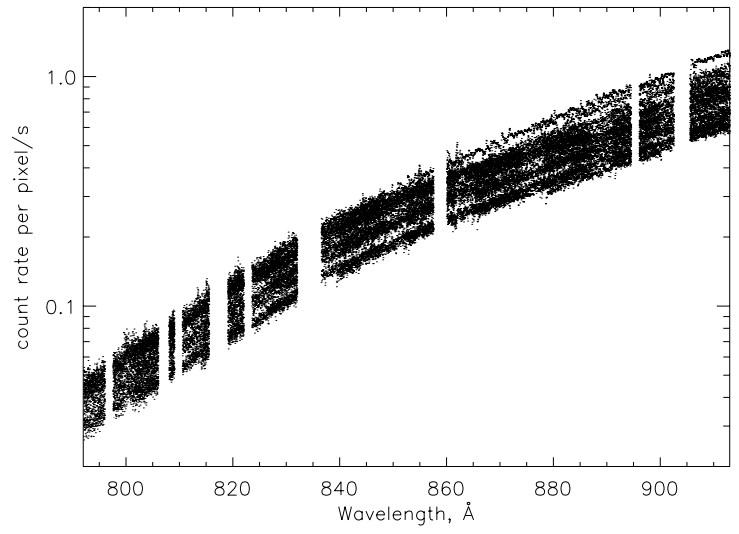

Fig. A.2. Quiet-Sun H I Lc data obtained with A detector in 1996.

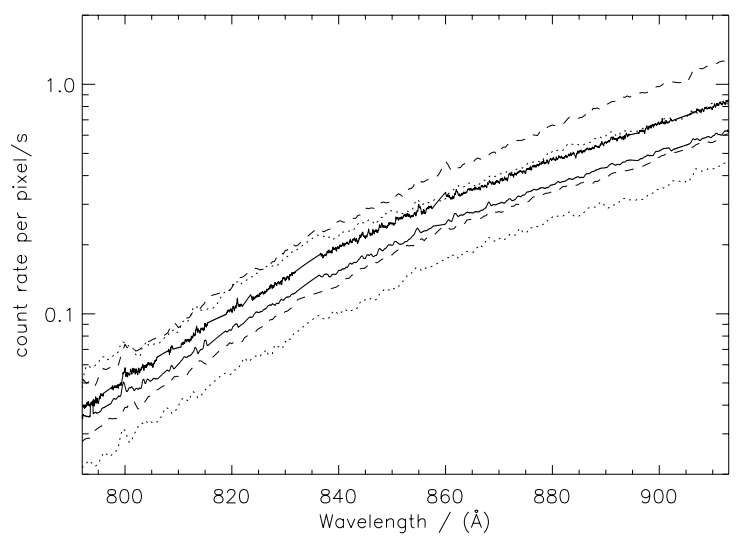

Fig. A.3. Quiet-Sun H I Lc data averaged values (solid line - upper curve) and highest and lowest curves (- -) obtained with A detector in 1996. Detector B data are overplotted (averaged values: solid line lower curve, highest and lowest curves: ...).

slit and the exposure time is performed. For each detector window the central part (coated with a $\mathrm{KBr}$ photocathode) is selected, and the data are combined to obtain the average spectrum between $792 \AA$ and $913 \AA$ for the $\mathrm{A}$ and $\mathrm{B}$ detector respectively. The following step consists in removing the spectral lines in this spectral range.

The results for the $14 \mathrm{H} \mathrm{I} \mathrm{Lc} \mathrm{spectra} \mathrm{recorded} \mathrm{in} \mathrm{the} \mathrm{quiet-}$ Sun areas with detector A are shown in Fig. A.2. Detector A and B data are interpolated, averaged and displayed in Fig. A.3, with the highest and lowest curves showing the variation of the quiet-Sun H I Lc.

The relative responsivity variations with wavelength of the detectors A and B are very similar in the H I Lc spectral range (with $\mathrm{KBr}$ photocathodes). This was expected since the optical path is the same as far as the scan mirror. Then, the differences of incidence on the scan mirror $\left(1.6^{\circ}\right)$ and on the grating $\left(3.2^{\circ}\right)$ have a small effect on the relative responsivity.

\section{A.2. Calibration}

The calibration was performed in January 1995 before delivery of SUMER for integration in the SOHO spacecraft. The results were published in Hollandt et al. (1996). In the vicinity of the H I Lc range the calibrated lines used are Ar II (740.3,

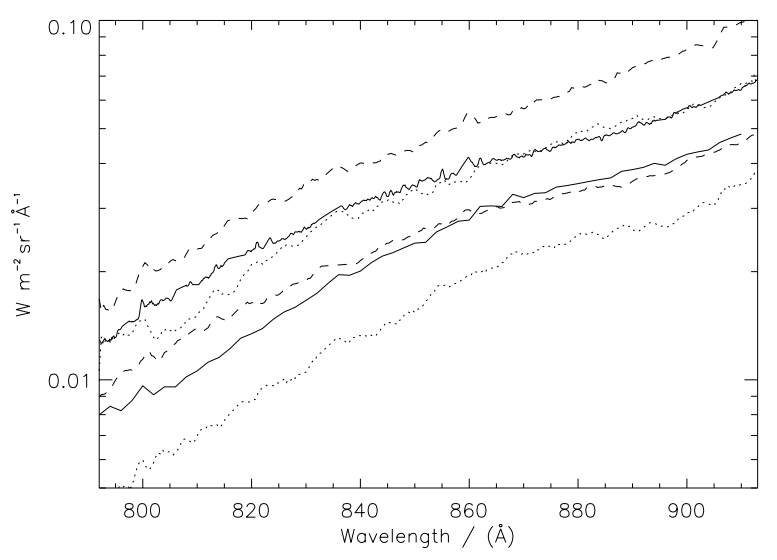

Fig. A.4. Quiet-Sun H I Lc calibrated data with radiometry.pro averaged values (solid line - upper curve) and highest and lowest curves (- -) obtained with A detector in 1996. Detector B data are overplotted (averaged values: solid line - lower curve, highest and lowest curves: ...).

744.9/745.3, 919.8, 932.1 $),$ Ar III (769.2 ̊̊), Ne I (740.3 $)$ and $\operatorname{Kr}$ II $(884.1 / 886.3,891.0,917.4,965.0 \AA)$. The spectral range of detector $\mathrm{A}$ in the first order begins near $780 \AA$. Shorter-wavelength lines are detected in the second grating order, but the efficiency in this (2nd order) wavelength domain is unrelated to the efficiency in the short (1st order) wavelength domain. To establish the calibration for H I Lc it is better to use the B detector in first order extending down to $670 \AA$ and to transfer the curve from B to A in the H I Lc spectral range where the calibration lines are sparse. Anyhow there is still a gap in the calibrated lines between $769 \AA$ and $884 \AA$ which introduces some uncertainties in the determination of the continuum slope.

The in-flight SUMER calibration with detector $A$ and detector B were respectively published by Wilhelm et al. (1997b) and Schühle et al. (2000). Radiometry.pro (which can be retrieved from sohowww.nascom.nasa.gov at /solarsoft/soho/sumer/idl/contrib/wilhelm/rad) for detectors $\mathrm{A}$ and $\mathrm{B}$ was used to provide the absolute calibration of the average HI Lc data shown in Fig. A.4 (radiometry . pro is updated from time to time to accommodate the latest calibration data). The $\mathrm{B}$ detector responsivity curve is similar to the one shown in the Curdt et al. (2001) atlas. For comparison, the HCO/SKYLAB data from average quiet Sun, network and cell (Vernazza \& Reeves (1978), used by Vernazza et al. (1981) to build VAL C, B and D semi-empirical atmospheric models) are also displayed in Fig. A.5.

There is a slight discrepancy in the H I Lc slope between both detectors. We will make the hypothesis that the B curve is the nearest to the true curve since the responsivity curve is constrained by the lines used during the pre-flight calibration below $800 \AA$. The B curve slope is very similar to the average quiet Sun slope observed by HCO/SKYLAB.

In Fig. A.6 the curves obtained from $8500 \mathrm{~K}, 9000 \mathrm{~K}$ and $10000 \mathrm{~K}$ colour temperatures are adjusted to fit the $\mathrm{B}$ detector calibrated data, with the average HCO/SKYLAB data for comparison. The quiet-Sun slopes at the head of H I Lc can be fitted by the colour temperature curves in the 
P. Lemaire et al.: Flare observation of the Sun as a star by SUMER/SOHO, Online Material $p 4$

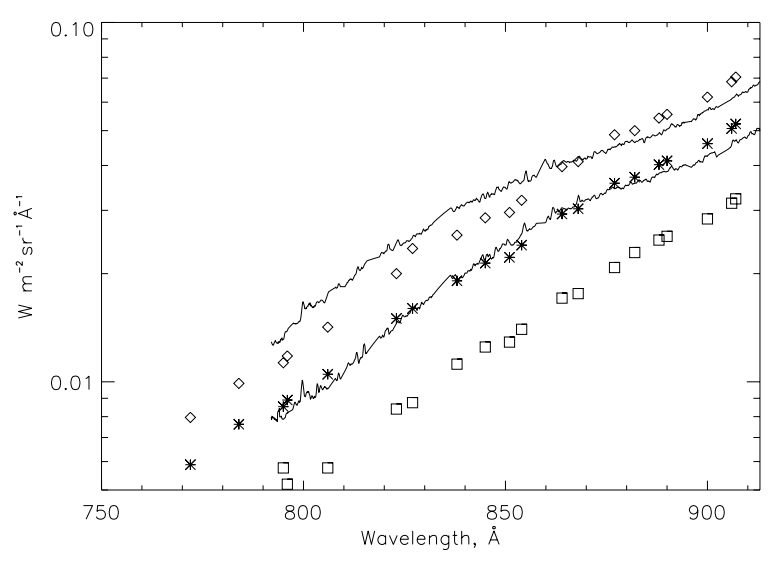

Fig. A.5. Quiet-Sun H I Lc calibrated data with radiometry .pro: detector A, average values (solid line, upper curve) and detector B, average values (solid line, lower curve). The data from $\mathrm{HCO} / \mathrm{SKYLAB}$ on average quiet Sun $(*)$, cell (square) and network (diamond) are given for comparison (Vernazza et al. 1981).

8500-10000 K temperature range, scaled to the data. This result is quite compatible with previous results obtained with HCO/SKYLAB data (Vernazza et al. 1981) and recently by Wilhelm \& Kalkofen (2003) using SUMER data.

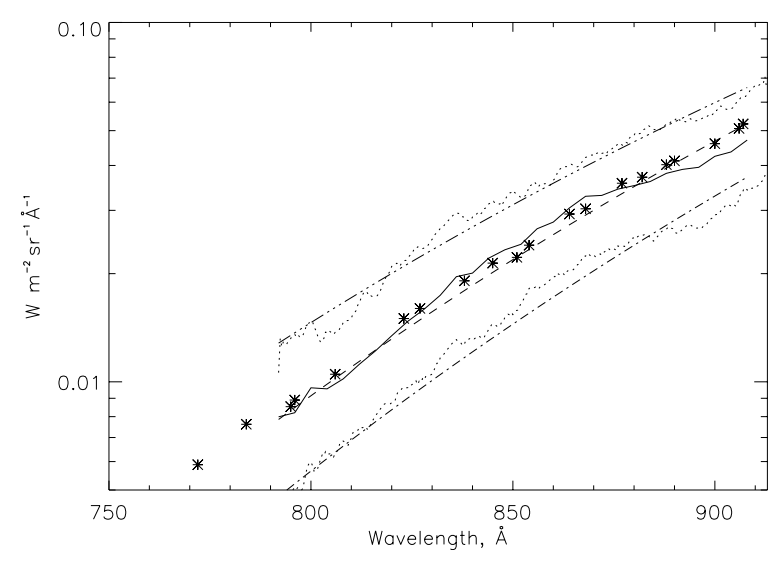

Fig. A.6. Quiet-Sun H I Lc calibrated data obtained with B detector and radiometry.pro, average values (solid line), highest and lowest curves (...). The data from HCO/SKYLAB on average quiet Sun $(*)$ are overplotted. The $8500 \mathrm{~K}(-.-), 9000 \mathrm{~K}(---)$ and $10000 \mathrm{~K}(-. .-)$ colour temperature curves are adjusted to the data.

There is still a discrepancy between the calibrated values of the H I Lc obtained with detectors A and B (separately calibrated) which needs further investigation. 